\title{
OSCILLATION AND NONOSCILLATION THEOREMS FOR SOME MIXED DIFFERENCE EQUATIONS
}

\author{
B. SMITH and W. E. TAYLOR, JR. \\ Department of Mathematics \\ Nabrit Science Center \\ Texas Southern University \\ Houston, Texas 77004
}

(Received October 10, 1989 and in revised form November 6, 1990)

\begin{abstract}
In this paper we investigate the oscillatory and nonoscillatory behavior of solutions of certain mixed third and fourth order difference equations. Specific results are also obtained for the constant coefficient cases.
\end{abstract}

KEY WORDS AND PHRASES: Mixed difference equations, third and fourth order equations, oscillatory and nonoscillatory solutions.

1991 AMS SUBJECT CLASSIFICATION CODES. 39A10, 39A12.

\section{INTRODUCTION.}

In this paper we study the oscillatory and nonoscillatory behavior of the solutions of certain third and fourth order difference equations. Until recently, excepting the studies by Cheng [1], Hooker and Patula [2], and [4, 5, 6, 7], there has not been much research devoted to the oscillation theory of difference equations of order greater than two.

For a sequence $U_{n}$, and a fixed real constant $a$, we define $\Delta_{a} U_{n}=U_{n+1}-a U_{n}$. When $a=1$ we shall write $\Delta U_{n}$ instead of $\Delta_{1} U_{n}$. We can define inductively $\Delta_{a}^{k} U_{n}-\Delta_{a}\left(\Delta_{a}^{k-1} U_{n}\right)$ for $k>1$. The operator $\Delta_{a}$ was introduced by J. Popenda [3] in his study of certain nonlinear second order difference equations.

The objects of this study will be the mixed difference equations

$$
\Delta^{2}\left(\Delta_{a} U_{n}\right)+(-1)^{i} P_{n} U_{n}=0 \quad i=1,2,
$$

and

$$
\Delta^{3}\left(\Delta_{a} U_{n}\right)+(-1)^{i} P_{n} U_{n}=0 \quad i=1,2,
$$

where $P_{n}$ is a sequence of positive numbers having a positive limit inferior, that is, there is a positive constant $c>0$ such that $P_{n}>c$ for all $n$ sufficiently large. We consider only nontrivial solutions. A solution is called nonoscillatory if it is eventually of constant sign (positive or negative) otherwise it is called oscillatory. The equations (1.1) and (1.2) are called mixed because of the two difference operators $\Delta$ and $\Delta_{a}$ appearing in the equations. 


\section{ASYMPTOTIC BEHAVIOR OF NONOSCILLATORY SOLUTIONS.}

In this section we study the asymptotic behavior of the nonoscillatory solutions of (1.1) and (1.2).

THEOREM 1. Suppose $U_{n}$ is a nonoscillatory solution of

$$
\Delta^{2}\left(\Delta_{a} U_{n}\right)+P_{n} U_{n}=0
$$

where $0<a \leq 1$. Then for all $n$ sufficiently large we have

$$
\operatorname{sgn} U_{n}=\operatorname{sgn} \Delta^{2} U_{n} \neq \operatorname{sgn} \Delta U_{n}=\operatorname{sgn} \Delta^{3} U_{n}
$$

and

$$
\lim _{n \rightarrow \infty} U_{n}=0 .
$$

PROOF. For $a=1$, Popenda and Schmeidel [4] have recently shown that (2.1) has a solution satisfying (2.2). A nonoscillatory solution may not exist if $0<a<1$, but if it does exist we show that it must satisfy (2.2) and (2.3). As the negative of a solution of equation (1.1) is also a solution of the same equation, it suffices to prove that an eventually positive solution of (2.1) satisfies (2.2). In this paper, we will assume that all inequalities about sequences hold for all $n$ sufficiently large. Let $U_{n}>0$ be a nonoscillatory solution of (2.1). Set

$$
Z_{n}=\Delta_{a} U_{n}=U_{n+1}-a U_{n}
$$

then by (2.1)

$$
\Delta^{2} Z_{n}=-P_{n} U_{n}<0
$$

so $\Delta Z_{n}$ is (eventually) strictly decreasing. From (2.5) it follows that if $\Delta Z_{n}$ is eventually negative we must have $Z_{n} \rightarrow-\infty$, however this is contradictory since $Z_{n}=U_{n+1}-a U_{n}=\Delta U_{n}+(1-a) U_{n} \rightarrow-\infty$ implies $\Delta U_{n} \rightarrow-\infty$, which forces $U_{n}$ to be eventually negative. We must have

$$
\Delta Z_{n}>0
$$

for all large $n$. Indeed we will show that $\lim _{n \rightarrow \infty} U_{n}=0$.

Writing (2.1) as $\Delta^{2} Z_{n}=-P_{n} U_{n}$ and summing from $N$ to $m-1$, where $N$ is chosen large enough so that $\Delta Z_{n}>0$ for all $n \geq N$, we get

$$
\Delta Z_{m}-\Delta Z_{N}=-\sum_{N}^{m-1} P_{n} U_{n}
$$

The $\lim$ inf condition on $P_{n}$ yields

$$
0<c \sum_{N}^{m-1} U_{n} \leq \sum_{N}^{m-1} P_{n} U_{n}<\Delta Z_{N} .
$$

Letting $m \rightarrow \infty$ we see that $\sum_{N}^{\infty} U_{n}<\infty$ and therefore $\lim _{n \rightarrow \infty} U_{n}=0$. Because $U_{n} \rightarrow 0$ as $n \rightarrow \infty$ it follows that $Z_{n} \rightarrow 0$ as $n \rightarrow \infty$. From (2.6), $Z_{n}$ is increasing, hence $Z_{n}<0$ eventually. It then follows from the inequality $Z_{n}=\Delta U_{n}+(1-a) U_{n}<0$ that $\Delta U_{n}<0$ and from (2.6) $\Delta Z_{n}=\Delta^{2} U_{n}+(1-a) \Delta U_{n}>0$ and thus $\Delta^{2} U_{n}>0$. Finally from (2.5), $\Delta^{2} Z_{n}=\Delta^{3} U_{n}+(1-a) \Delta^{2} U_{n}<0$ and we get $\Delta^{3} U_{n}<0$ and the proof is complete.

Our next result though similar to the previous one requires $a>1$.

THEOREM 2. Consider the following equation

$$
\Delta^{2}\left(\Delta_{a} U_{n}\right)-P_{n} U_{n}=0
$$


where $a>1$. If $U_{n}$ is a nonoscillatory solution of (2.7) then for all $n$ sufficiently large

$$
\operatorname{sgn} U_{n}=\operatorname{sgn} \Delta U_{n}=\operatorname{sgn} \Delta^{2} U_{n},
$$

and

$$
\lim _{n \rightarrow \infty}\left|U_{n}\right|=\lim _{n \rightarrow \infty}\left|\Delta U_{n}\right|=\lim _{n \rightarrow \infty}\left|\Delta^{2} U_{n}\right|=\infty .
$$

PROOF. Assume without loss of generality that $U_{n}>0$ for all $n$ sufficiently large. Set

$$
Z_{n}=\Delta_{a} U_{n}=U_{n+1}-a U_{n},
$$

then by (2.7)

$$
\Delta^{2} Z_{n}=P_{n} U_{n}>0 .
$$

So $\Delta Z_{n}$ is increasing. If $\Delta Z_{n}$ is eventually positive then as $n \rightarrow \infty, Z_{n} \rightarrow \infty$ and since $Z_{n}=\Delta U_{n}+(1-a) U_{n}$ and $a>1$ it follows that $\Delta U_{n} \rightarrow \infty$, which in turn implies $U_{n} \rightarrow \infty$. To see why $\Delta^{2} U_{n} \rightarrow \infty$, note that $U_{n} \rightarrow \infty$ implies $\Delta^{2} Z_{n} \rightarrow \infty$ and $\Delta Z_{n} \rightarrow \infty$ because of (2.8). But $\Delta Z_{n}=\Delta^{2} U_{n}+(1-a) \Delta U_{n}$ and the result follows. Now, if $\Delta Z_{n}$ were eventually negative and increasing then $\Delta Z_{n}$ would have a limit as $n \rightarrow \infty$. However $\Delta Z_{n}$ having a limit implies that $\Sigma^{\infty} U_{n}<\infty$ and this implies $U_{n} \rightarrow 0$. But $U_{n} \rightarrow 0$ implies $Z_{n} \rightarrow 0$ also and therefore since $Z_{n}$ is decreasing to zero, $Z_{n}>0$. But $Z_{n}=\Delta U_{n}+(1-a) U_{n}>0$ implies $\Delta U_{n}>0$, a contradiction since $U_{n}>0$ and $\Delta U_{n}>0$ is inconsistent with $U_{n} \rightarrow 0$. Hence (2.7) cannot have a nonoscillatory solution with $\Delta Z_{n} \Delta^{2} Z_{n}<0$ for all $n$ sufficiently large.

It should be noted that the condition $0<a<1$ was crucial in the proof of Theorem 1. Our next result requires $a>1$ and is similar to one obtained in [2] for the equation

$$
\Delta^{4} U_{n}-P_{n+2} U_{n+2}=0 \text {. }
$$

THEOREM 3. Consider the equation

$$
\Delta^{3}\left(\Delta_{a} U_{n}\right)-P_{n} U_{n}=0
$$

where $a \geq 1$. If $U_{n}$ is a nonoscillatory solution of (2-) then for all $n$ sufficiently large either

$$
\operatorname{sgn} U_{n}=\operatorname{sgn} \Delta U_{n}=\operatorname{sgn} \Delta^{2} U_{n}=\operatorname{sgn} \Delta^{3} U_{n}
$$

or

$$
\left.\operatorname{sgn} U_{n}=\operatorname{sgn} \Delta_{(} \Delta_{a} U_{n}\right)=\operatorname{sgn} \Delta_{a} U_{n}=\operatorname{sgn} \Delta^{2}\left(\Delta_{a} U_{n}\right) .
$$

PROOF. We prove the case for $a>1$. The proof for $a=1$ is similar. There is no loss of generality in assuming $U_{n}$ is an eventually positive solution of (2.9). Set $Z_{n}-\Delta_{a} U_{n}-U_{n+1}-a U_{n}$. Then by (2.9)

$$
\Delta^{3} Z_{n}=P_{n} U_{n}>0 \text {. }
$$

Clearly $\Delta^{2} Z_{n}$ is increasing. In case $\Delta^{2} Z_{n}$ is eventually positive we will have $\lim _{n \rightarrow \infty} \Delta Z_{n}=\lim _{n \rightarrow \infty} Z=\infty$, and since $Z_{n}<U_{n+1}$ it follows that $U_{n} \rightarrow \infty$. Since $P_{n}>c$ for large $n$

$$
\lim _{n \rightarrow \infty} \Delta^{3} Z_{n}=\lim _{n \rightarrow \infty} \Delta^{2} Z_{n}=\infty \text {. }
$$

Since $Z_{n}=\Delta U_{n}+(1-a) U_{n} \rightarrow \infty$ and $a>1$ it follows that $\Delta U_{n} \rightarrow \infty$. Examining $\Delta Z_{n}=\Delta^{2} U_{n}+(1-a) \Delta U_{n}$ we see that $\Delta^{2} U_{n} \rightarrow \infty$ as $n \rightarrow \infty$. Continuing in this manner we see that (I) holds eventually.

Next we consider the case where $\Delta^{3} Z_{n}>0$ and $\Delta^{2} Z_{n}<0$. Then $\lim _{n \rightarrow \infty} \Delta^{2} Z_{n}$ exists and summing (2.10) from $N$ to $m-1$ yields 


$$
-\Delta^{2} Z_{N}>\Delta^{2} Z_{m}-\Delta^{2} Z_{N}=\sum_{N}^{m-1} P_{n} U_{n} \geq c \sum_{N}^{m-1} U_{n}
$$

Letting $m \rightarrow \infty$ it then follows that $\sum_{N}^{\infty} U_{n}<\infty$ and hence $\lim _{n \rightarrow \infty} U_{n}=0$ which implies $Z_{n} \rightarrow 0$. Thus if $\Delta^{3} Z_{n}>0$ and $\Delta^{2} Z_{n}<0$ then eventually we must have

$$
\Delta^{3} Z_{n}>0, \Delta^{2} Z_{n}<0, \Delta Z_{n}>0,
$$

because $\Delta^{2} Z_{n}<0$ and $\Delta Z_{n}<0$ is inconsistent with $Z_{n} \rightarrow 0$, it then follows that either (i) $Z_{n}>0$ or (ii) $Z_{n}<0$ eventually. We will show that (i) is impossible. If (i) held then since $Z_{n}=\Delta U_{n}+(1-a) U_{n}>0$ it follows that $\Delta U_{n}>0$, in fact we have that $\Delta U_{n}>k+(a-1) U_{n}>k$ for some positive constant $k$ and so $U_{n} \rightarrow \infty$ as $n \rightarrow \infty$. But this implies $\Delta^{3} Z_{n} \rightarrow \infty$, so we must have $\Delta^{2} Z_{n}>0$ eventually, contradicting (2.11). So (i) cannot hold, resulting in (ii) holding eventually.

\section{SUFFICIENT CONDITIONS FOR OSCILLATION AND/OR NONOSCILLATION.}

THEOREM 4. Every nontrivial bounded solution of

$$
\Delta^{3}\left(\Delta_{a} U_{n}\right)+P_{n} U_{n}=0
$$

where $a>1$, is oscillatory.

PROOF. Suppose (3.1) has a bounded nonoscillatory solution $U_{n}$ satisfying $U_{n}>0$ for large $n$. Letting $Z_{n}=\Delta_{a} U_{n}=U_{n+1}-a U_{n}$, we see that $Z_{n} \geq-a U_{n}$. From (3.1), $\Delta^{3} Z_{n}=-P_{n} U_{n}<0$. Obviously $\Delta^{2} Z_{n}$ is decreasing, and if $\Delta^{2} Z_{n}$ is eventually negative, we see that $Z_{n} \rightarrow \infty$. This clearly contradicts the boundedness of $U_{n}$. Thus, we consider the case where $\Delta^{2} Z_{n}>0$. In this case $\lim _{n \rightarrow \infty} \Delta^{2} Z_{n}=t \geq 0$. Using the fact $P_{n}$ is bounded away from zero for large $n$, it follows that $\Delta Z_{n}<0$ and $Z_{n}>0$ for large $n$. Furthermore, $\lim _{n \rightarrow \infty} U_{n}=0$, since $\Delta^{2} Z_{n} \rightarrow t$ implies $\sum^{\infty} U_{n}<\infty$. Since $a>1$ and

$$
Z_{n}=\Delta U_{n}+(1-a) U_{n}>0,
$$

$\Delta U_{n}>0$ for all $n$ sufficiently large. But this is a contradiction, since $U_{n} \Delta U_{n}>0$ is incongruent with $U_{n} \rightarrow 0$.

EXAMPLE. The equation

$$
\Delta^{3}\left(\Delta_{2} U_{n}\right)+\frac{1}{16} U_{n}=0
$$

has the sequence $U_{n}=\left(\frac{3}{2}\right)^{n}$ as a solution. Hence equation (3.1) may have nonoscillatory solutions.

Before stating our final results consider the constant coefficient case $P_{n}=Q, Q>0$.

Equation (1.1) with $P_{n}=Q$ is

$$
\Delta^{3} U_{n}+(1-a) \Delta^{2} U_{n}+(-1)^{i} Q U_{n}=0 \quad i=1,2 .
$$

So the characteristic polynomial is

$$
f(t)=(t-1)^{3}+(1-a)(t-1)^{2}+(-1)^{i} Q \quad i=1,2 .
$$

Similarly, equation (1.2) can be written as

$$
\Delta^{4} U_{n}+(1-a) \Delta^{3} U_{n}+(-1)^{i} Q U_{n}=0 \quad i=1,2
$$

with characteristic polynomial

$$
g(t)=(t-1)^{4}+(1-a)(t-1)^{3}+(-1)^{i} Q \quad i=1,2 .
$$


The proofs of our final results follow from a careful examination of the characteristic polynomials $\left(C_{1}\right)$ and $\left(C_{2}\right)$.

THEOREM 5. Consider (2.4) where $0<a<1, P_{n}-Q$ constant and $Q>a$, then all nontrivial solutions of (2.4) are oscillatory.

EXAMPLE. The general solution of

$$
\Delta^{2}\left(\Delta_{1 / 2} U_{n}\right)+6 U_{n}=0
$$

is

$$
U_{n}=K_{1}(-1)^{n}+K_{2}(11 / 2)^{n / 2} \sin n \varnothing+K_{3}(11 / 2)^{n / 2} \cos n \varnothing
$$

where $\varnothing=\arctan (\sqrt{39} / 7)$ it follows from Theorem 1 or from Theorem 5 that all solutions of (3.4) are oscillatory.

THEOREM 6. Consider (2.7) where $0<a<1, Q=\frac{R^{3}}{64}$ constant and $0<a<1-\frac{3}{4} R$, then all solutions of (2.7) are nonoscillatory.

THEOREM 7. Consider (2.9) where $0<a<1, P_{n}=Q$ constant and $Q>0$, then (2.9) has oscillatory and nonoscillatory solutions. Moreover, all nonoscillatory solutions are bounded and converge to zero.

Note that when $a=1, P_{n}=Q$ constant, equation (2.7) becomes $\Delta^{3} U_{n}-Q U_{n}=0$. Clearly this equation has oscillatory solutions for any $Q>0$. Thus, the result of Theorem 6 depends upon $0<a<1$. Furthermore, it should be noted that Theorem 7 is interesting because, when $a>1$ and $P_{n}$ is constant (2.7) must have an unbounded nonoscillatory solution. Clearly the boundedness of the nonoscillatory solutions can be attributed to the parameter $a$ in the operator $\Delta_{a}$.

\section{REFERENCES}

1. CHENG, S. S., On a Class of Fourth Order Linear Recurrence Equations, Internat. J. Math. and Math. Sci., I (1984), 131-149.

2. HOOKER, J. W. and PATULA, W. T., Growth and Oscillation Properties of Solutions of a Fourth Order Linear Difference Equation, J. Austral. Math. Soc. Ser, B26 (1985), No. 3, 310-328.

3. POPENDA, J., Oscillation and Nonoscillation Theorems for Second Order Difference Equations, $\mathbf{L}$ Math. Anal. Apple 123 (1987), 34-38.

4. POPENDA, J. and SCHMEIDEL, E., Nonoscillatory Solutions of Third Order Difference Equations, preprint.

5. SMITH, B. and TAYLOR, JR., W. E., Oscillatory and Asymptotic Behavior of Certain Fourth Order Difference Equations, Rocky Mt. J. Math., 16 (1986), 403-406.

6. SMITH, B., Oscillation and Nonoscillation Theorems for Third Order Quasi-Adjoint Difference Equations, Port. Math. 45 (1988), 229-243.

7. TAYLOR, JR., W. E., Oscillation Properties of Fourth Order Difference Equations, Port. Math. 45 (1988), 105-114. 


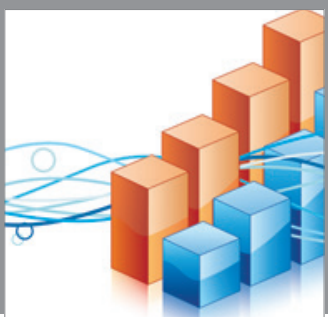

Advances in

Operations Research

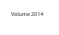

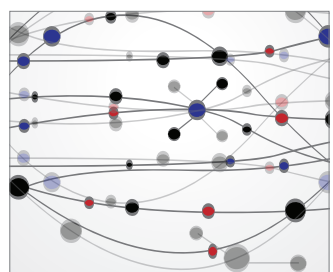

\section{The Scientific} World Journal
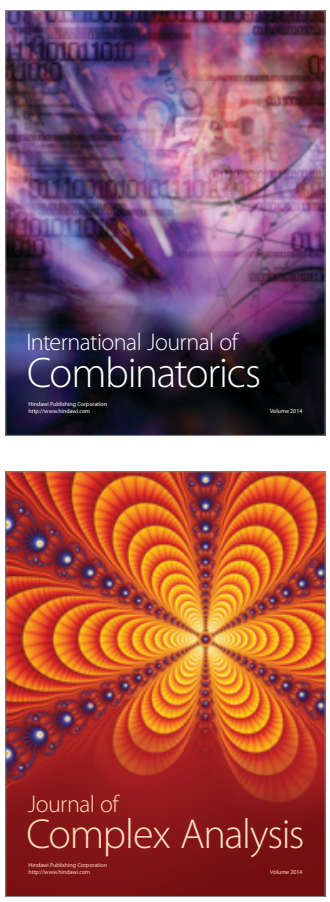

International Journal of

Mathematics and

Mathematical

Sciences
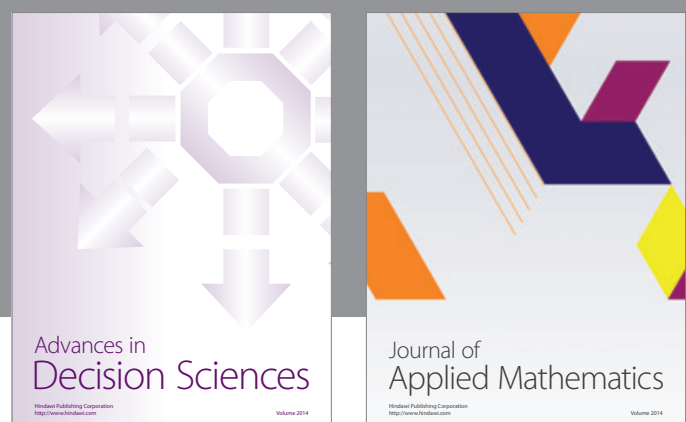

Journal of

Applied Mathematics
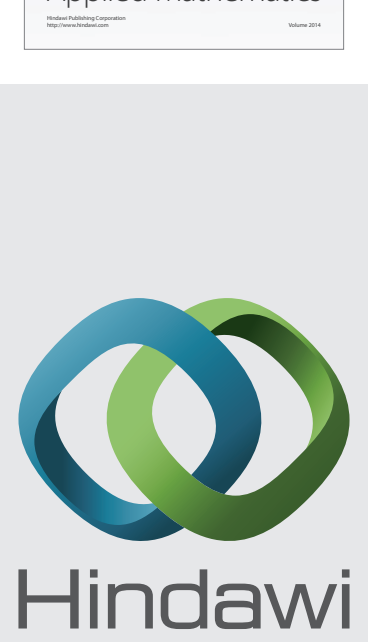

Submit your manuscripts at http://www.hindawi.com
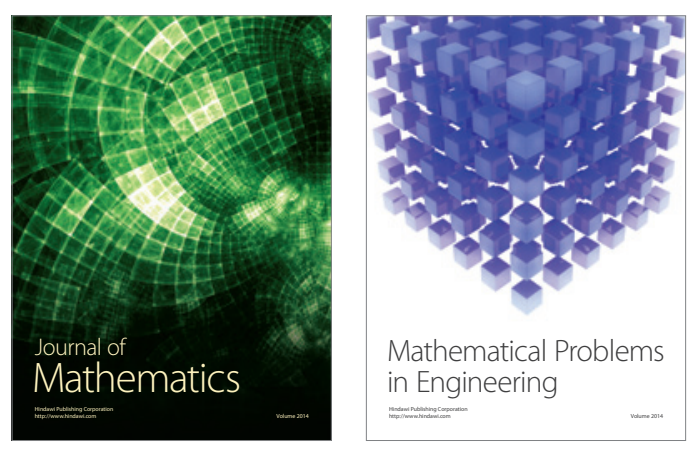

Mathematical Problems in Engineering
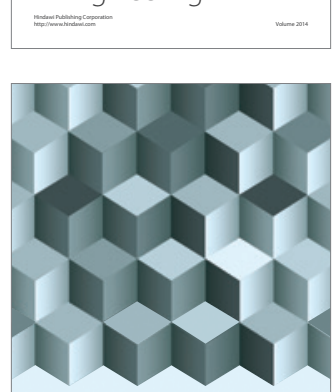

Journal of

Function Spaces
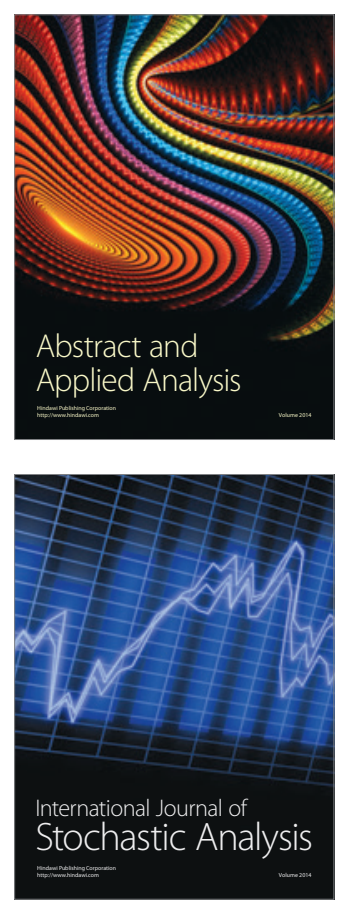

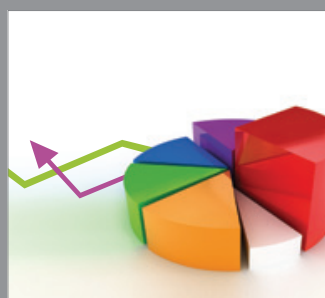

ournal of

Probability and Statistics

Promensencen
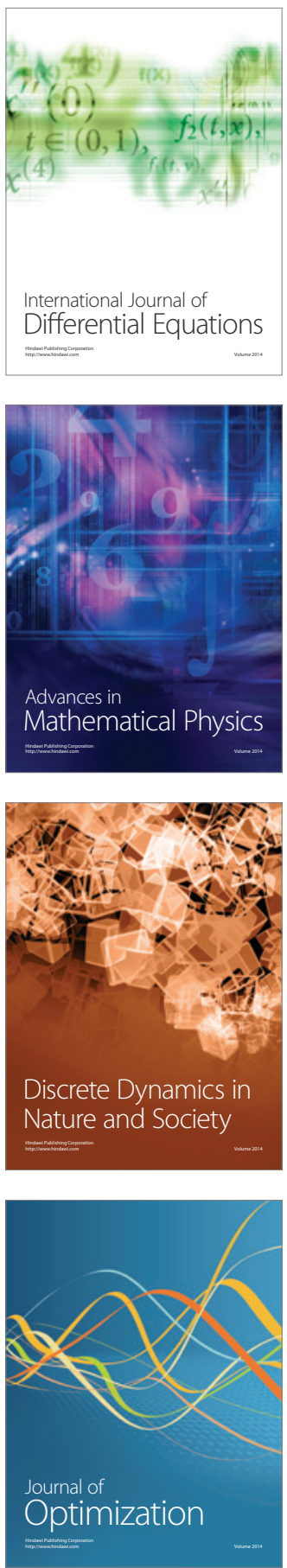